PROCEEDINGS OF THE

AMERICAN MATHEMATICAL SOCIETY

Volume 125, Number 2, February 1997, Pages 603-609

S $0002-9939(97) 03855-0$

\title{
HYPERSPACES AND OPEN MONOTONE MAPS OF HEREDITARILY INDECOMPOSABLE CONTINUA
}

\author{
MICHAEL LEVIN
}

(Communicated by James West)

\begin{abstract}
We prove the following theorems:
Theorem 1. Let $X$ be an $n$-dimensional hereditarily indecomposable continuum. Then there exist 1-dimensional hereditarily indecomposable continua $Y_{1}, Y_{2}, \ldots, Y_{n}$ and monotone maps $p_{i}: X \longrightarrow Y_{i}$ such that $\left(p_{1}, p_{2}, \ldots, p_{n}\right)$ : $X \longrightarrow Y_{1} \times Y_{2} \times \ldots \times Y_{n}$ is an embedding and the space $\mathcal{C}(X)$ of all subcontinua of $X$ is embeddable in $\mathcal{C}\left(Y_{1}\right) \times \mathcal{C}\left(Y_{2}\right) \times \ldots \times \mathcal{C}\left(Y_{n}\right)$ by $K \in \mathcal{C}(X) \longrightarrow$ $\left(p_{1}(K), p_{2}(K), \ldots, p_{n}(K)\right)$.

Theorem 2. For every open monotone map $\varphi$ with non-trivial sufficiently small fibers on a finite dimensional hereditarily indecomposable continuum $X$ with $\operatorname{dim} X \geq 2$ there exists a 1-dimensional subcontinuum $Y \subset X$ such that $\operatorname{dim} \varphi(Y)=\infty$ and the restriction of $\varphi$ to $Y$ is also monotone and open.

The connection between these theorems and other results in Hyperspace theory is studied.
\end{abstract}

\section{INTRODUCTION}

Let $X$ be a compact metrizable space. $2^{X}$ denotes the space of closed subsets of $X$ endowed with the Hausdorff metric. $2^{X}$ is compact. $\mathcal{C}(X)$ is the closed subset of $2^{X}$ that consists of the subcontinua of $X$ and $\mathcal{C}_{\epsilon}(X), \epsilon \geq 0$, is the closed subset of $\mathcal{C}(X)$ which consists of the subcontinua of $X$ with diam $\leq \epsilon$. By the hyperspace of $X$ we mean $\mathcal{C}(X)$.

In Section 2 we prove

Theorem 1.1. Let $X$ be an $n$-dimensional hereditarily indecomposable continuum. Then there exist 1-dimensional hereditarily indecomposable continua $Y_{1}, Y_{2}, \ldots, Y_{n}$ and monotone maps $p_{i}: X \longrightarrow Y_{i}$ such that $\left(p_{1}, p_{2}, \ldots, p_{n}\right): X \longrightarrow Y_{1} \times Y_{2} \times \ldots \times Y_{n}$ is an embedding and $\mathcal{C}(X)$ is embeddable in $\mathcal{C}\left(Y_{1}\right) \times \mathcal{C}\left(Y_{2}\right) \times \ldots \times \mathcal{C}\left(Y_{n}\right)$ by $K \in$ $\mathcal{C}(X) \longrightarrow\left(p_{1}(K), p_{2}(K), \ldots, p_{n}(K)\right)$.

Theorem 1.2. Let $X$ be a finite dimensional hereditarily indecomposable continuum of $\operatorname{dim} \geq 2$ and let $\varphi$ be an open monotone map on $X$ with non-trivial sufficiently small fibers. Then there exists a 1-dimensional subcontinuum $Y$ of $X$ such that $\operatorname{dim} \varphi(Y)=\infty$.

The restriction of $\varphi$ to $Y$ is also open and monotone. This follows from

Received by the editors January 1, 1995

1991 Mathematics Subject Classification. Primary 54B20, 54F15, 54F45.

Key words and phrases. Hyperspaces, hereditarily indecomposable continua, open monotone maps. 
Proposition 1.3. Let $X$ be a hereditarily indecomposable continuum and let $\varphi$ be a monotone map on $X$. Then for every subcontinuum $Y$ of $X$ we have either $Y=\varphi^{-1}(\varphi(Y))$ or $\varphi(Y)$ is a singleton.

Proof. Assume that $\varphi(Y)$ is not a singleton. For every $y \in \varphi(Y), \varphi^{-1}(y)$ is a continuum which intersects $Y$, and since $Y$ is not contained in $\varphi^{-1}(y)$ we have $\varphi^{-1}(y) \subset Y$ and hence $Y=\varphi^{-1}(\varphi(Y))$.

Whitney maps give a tool for constructing monotone open maps on hereditarily indecomposable continua.

Definition 1.4. (See [9]) Let $X$ be a continuum. $A$ map $W: \mathcal{C}(X) \longrightarrow \mathbb{R}^{+}$is called a Whitney map if $W$ vanishes on the set of singletons in $\mathcal{C}(X)$ and if $A \neq B$ in $\mathcal{C}(X)$ and $A \subset B$ implies $W(A)<W(B)$.

Whitney maps always exist: if $\left\{f_{n}\right\}_{n=1}^{\infty}$ is a dense sequence of functions in $C(X,[0,1])$ and $W_{n}(A)=\operatorname{diam} f_{n}(A), W_{n}: \mathcal{C}(X) \longrightarrow[0,1]$, then $W=\sum_{n=1}^{\infty} W_{n} / 2^{n}$ is a Whitney map.

If $X$ is hereditarily indecomposable, then for every $0<t \leq W(X), W^{-1}(t)$ is an upper and lower semicontinuous decomposition of $X$ and the corresponding quotient map is an open monotone map with non-trivial fibers which can be made arbitrarily small by choosing $t$ close to 0 . Note that if $\varphi$ is a monotone open map on $X$, then $\mathcal{C}(\varphi(X))$ can be embedded in $\mathcal{C}(X)$ by $K \in \mathcal{C}(\varphi(X)) \longrightarrow \varphi^{-1}(K) \in \mathcal{C}(X)$.

It is well-known (Bing [1]) that every $n$-dimensional continuum contains an $(n-1)$-dimensional hereditarily indecomposable subcontinuum. Applying Theorem 1.2 we obtain

Theorem 1.5. Every finite dimensional hereditarily indecomposable continuum $X$ with $\operatorname{dim} X \geq 2$ (and so every finite dimensional continuum $X$ of $\operatorname{dim} \geq 3$ ) contains a 1-dimensional subcontinuum $Y$ such that $\operatorname{dim} \mathcal{C}(Y)=\infty$.

In [3] Kelley proved that if $X$ is a hereditarily indecomposable continuum of $\operatorname{dim} \geq 2$, then the image of $X$ under an open monotone map with sufficiently small non-trivial fibers is infinite dimensional and hence $\operatorname{dim} \mathcal{C}(X)=\infty$. So Theorems 1.2 and 1.5 generalize this result of Kelley. Recently it has been proved that the hyperspace of every 2-dimensional continuum is infinite dimensional; see [7].

Question 1.6. Does Theorem 1.5 hold for every 2-dimensional continuum $X$ ?

It seems that this question is still open. The following theorem gives a partial answer to Question 1.6.

Theorem 1.7. (Levin and Sternfeld [6]) For each natural number $n$ every 2-dimensional continuum $X$ contains a 1-dimensional subcontinuum $Y_{n}$ with $\operatorname{dim} \mathcal{C}\left(Y_{n}\right) \geq n$.

Note that from Theorem 1.5 it follows that there exist 1-dimensional hereditarily indecomposable continua with infinite dimensional hyperspaces. Clearly the same result can also be obtained from Theorem 1.1. The first examples of such continua were given by Lewis [8]. (This should be compared with Theorem 1.9.)

In Section 2 we present a few applications of the approach of this note. We will also use

Theorem 1.8. (Bing [1]) An n-dimensional hereditarily indecomposable continuum contains a point $p$ such that every non-trivial subcontinuum containing $p$ is of dimension $n$. 
Theorem 1.9. (Krasinkiewicz [4]) The image of a planar hereditarily indecomposable continuum under an open monotone map is of dimension $\leq 1$. The hyperspace of a planar non-trivial hereditarily indecomposable continuum is 2-dimensional.

Theorem 1.10. (Kelley [3]) Let $X$ be a continuum and let $\varphi$ be an open monotone map on $X$ with non-trivial fibers and $\operatorname{dim} \varphi(X)<\infty$. Then there exists a 0 dimensional closed subset $Y$ of $X$ such that $\varphi(Y)=\varphi(X)$.

\section{Proofs AND APPlications}

In this section we prove Theorem 1.1 and Proposition 2.1 (a weaker version of Theorem 1.2) which is the first step in proving Theorem 1.2, we present some applications of the proofs of Theorem 1.2 and Proposition 2.1, and finally we prove Theorem 1.2.

Recall the following facts from Dimension Theory [2]. Let $f: X \longrightarrow Y . \operatorname{dim} f=$ $\sup \left\{\operatorname{dim} f^{-1}(y): y \in Y\right\}$. 0-dimensional maps are also called light. For every $n$ dimensional compactum and for every $0 \leq k \leq n$ there exists an $(n-k)$-dimensional map $g: X \longrightarrow \mathbb{R}^{k}$. If $X$ is an $n$-dimensional compactum, then there exists $\epsilon>0$ such that the image of $X$ under every $\epsilon$-map (=map with fibers of diam $\leq \epsilon$ ) is of $\operatorname{dim} \geq n$.

Proof of Theorem 1.1. The proof is based on the ideas of [5]. Take a 0-dimensional map $g=\left(g_{1}, g_{2}, \ldots, g_{n}\right): X \longrightarrow \mathbb{R}^{n}$ where $g_{i}: X \longrightarrow \mathbb{R}$. Let $g_{i}=h_{i} \circ p_{i}$ be the monotone-light decomposition of $g_{i}$ where $p_{i}$ is monotone and $h_{i}$ is light. Denote $Y_{i}=p_{i}(X)$. Since $h_{i}$ maps $Y_{i}$ to $\mathbb{R}$, by Hurewicz's theorem $\operatorname{dim} Y_{i} \leq 1$. Define $p=\left(p_{1}, p_{2}, \ldots, p_{n}\right): X \longrightarrow Y_{1} \times Y_{2} \times \ldots \times Y_{n}$. Clearly for every $x \in X, p^{-1}(p(x))=$ $p_{1}^{-1}\left(p_{1}(x)\right) \cap p_{2}^{-1}\left(p_{2}(x)\right) \cap \ldots \cap p_{n}^{-1}\left(p_{n}(x)\right)$. So $p$ is monotone (as $X$ is hereditarily indecomposable and $p_{i}$ are monotone) and since the fibers of $p$ are contained in the fibers of $g, p$ has to be an embedding. Define $p_{i}^{*}: \mathcal{C}(X) \longrightarrow \mathcal{C}\left(Y_{i}\right)$ by $K \in \mathcal{C}(X) \longrightarrow$ $p_{i}(K) \in \mathcal{C}\left(Y_{i}\right)$. Obviously $p_{i}^{*}$ are continuous.

Let us show that $p^{*}=\left(p_{1}^{*}, p_{2}^{*}, \ldots, p_{n}^{*}\right): \mathcal{C}(X) \longrightarrow \mathcal{C}\left(Y_{1}\right) \times \mathcal{C}\left(Y_{2}\right) \times \ldots \times \mathcal{C}\left(Y_{n}\right)$ is an embedding. Take $K_{1}, K_{2} \in \mathcal{C}(X)$ such that $p_{i}\left(K_{1}\right)=p_{i}\left(K_{2}\right)$ for every $i$. Denote $T_{i}=p_{i}\left(K_{1}\right)=p_{i}\left(K_{2}\right)$. If for some $i, T_{i}$ is not a singleton, then by Proposition 1.3 $K_{1}=K_{2}=p_{i}^{-1}\left(T_{i}\right)$. If for every $i, T_{i}$ is a singleton, then $K_{1}=K_{2}$ as $p$ is an embedding. So $p^{*}$ is an embedding.

Proposition 2.1. Let $X$ be a finite dimensional hereditarily indecomposable continuum and let $f$ be an open monotone map of $X$ with sufficiently small nontrivial fibers. Then there exists a 1-dimensional subcontinuum $Y$ of $X$ such that $\operatorname{dim} f(Y) \geq \operatorname{dim} X$.

Proof. Let $n=\operatorname{dim} X$, let $g: X \longrightarrow \mathbb{R}^{n-1}$ be a 1-dimensional map and let $g=h \circ p$ be the monotone-light decomposition of $g$ where $p$ is monotone and $h$ is light. Clearly $p$ is 1 -dimensional. As $h: p(X) \longrightarrow \mathbb{R}^{n-1}$ is light, by Hurewicz's theorem $\operatorname{dim} p(X) \leq n-1$.

Define $q: X \longrightarrow p(X) \times f(X)$ by $q(x)=(p(x), f(x))$ and let $Z=q(X)$. For every $x \in X, q^{-1}(q(x))=p^{-1}(p(x)) \cap f^{-1}(f(x))$. As $X$ is hereditarily indecomposable and $p$ and $f$ are monotone, every fiber of $q$ coincides with a fiber of $p$ or with a fiber of $f$.

Denote $X_{p}=\left\{x \in X: q^{-1}(q(x))=p^{-1}(p(x))\right\}$ and $X_{f}=\left\{x \in X: q^{-1}(q(x))=\right.$ $\left.f^{-1}(f(x))\right\}$. Clearly $X=X_{p} \cup X_{f}$ and $X_{f}=f^{-1}\left(f\left(X_{f}\right)\right)=q^{-1}\left(q\left(X_{f}\right)\right), X_{p}=$ $p^{-1}\left(p\left(X_{p}\right)\right)=q^{-1}\left(q\left(X_{p}\right)\right)$. 
$X_{f}$ is closed in $X$. Indeed, let $x_{1}, x_{2}, \ldots \in X_{f}$ converge to $x \in X$. Define $K_{i}^{f}=f^{-1}\left(f\left(x_{i}\right)\right), K_{i}^{p}=p^{-1}\left(p\left(x_{i}\right)\right)$ and $K^{f}=f^{-1}(f(x)), K^{p}=p^{-1}(p(x))$. As $x_{i} \in X_{f}, K_{i}^{f} \subset K_{i}^{p}$. Since $f$ is open, $K^{f}=\lim K_{i}^{f}$ in $2^{X}$ and as $p$ is continuous we have $K^{f} \subset K^{p}$, that is, $x \in X_{f}$.

$X_{f}$ is 1-dimensional. Indeed, for every $x \in X_{f}, f^{-1}(f(x)) \subset X_{f} \cdot f^{-1}(f(x))$ is non-trivial and 1-dimensional since $f^{-1}(f(x)) \subset p^{-1}(p(x))$ and $\operatorname{dim} p^{-1}(p(x))$ $=1$ as $p$ is 1-dimensional. Take any component $T$ of $X_{f}$. For every $x \in T$, $x \in f^{-1}(f(x)) \subset T$ and hence by Theorem $1.8 \operatorname{dim} T=1$. So $\operatorname{dim} X_{f}=1$.

$q$ acts on $X_{p}$ as $p$ and on $X_{f}$ as $f$. By this we mean the following. Let $q_{p}$ and $q_{f}$ be the projections of $Z$ to $p(X)$ and $f(X)$ respectively. Clearly $p=q_{p} \circ q$ and $f=q_{f} \circ q$.

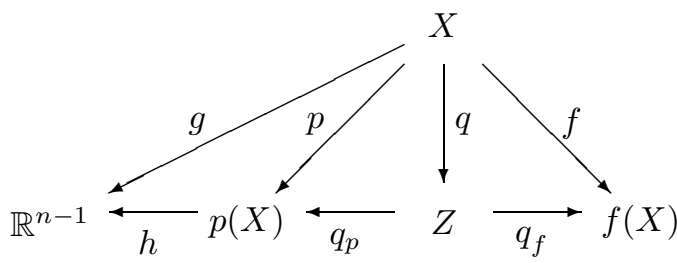

It is easy to see that $q\left(X_{p}\right)=\left\{z \in Z: z=q_{p}^{-1}\left(q_{p}(z)\right)\right\}, q\left(X_{f}\right)=\{z \in Z$ : $\left.z=q_{f}^{-1}\left(q_{f}(z)\right)\right\}$ and hence $q_{p}$ and $q_{f}$ are homeomorphisms on $q\left(X_{p}\right)$ and $q\left(X_{f}\right)$ respectively.

So $\operatorname{dim} q\left(X_{p}\right)=\operatorname{dim} p\left(X_{p}\right) \leq \operatorname{dim} p(X)=n-1$ and $\operatorname{dim} q\left(X_{f}\right)=\operatorname{dim} f\left(X_{f}\right)$. The fibers of $q$ are contained in the fibers of $f$ and we can assume that the fibers of $f$ are so small that $\operatorname{dim} Z \geq n$. Since $X_{f}$ is closed, $n \leq \operatorname{dim} Z=$ $\max \left\{\operatorname{dim} q\left(X_{p}\right), \operatorname{dim} q\left(X_{f}\right)\right\} \leq \max \left\{n-1, \operatorname{dim} f\left(X_{f}\right)\right\}$ and hence $\operatorname{dim} f\left(X_{f}\right) \geq n$. Take a component $T$ of $f\left(X_{f}\right)$ such that $\operatorname{dim} T \geq n$ and set $Y=f^{-1}(T)$. Then $Y$ is a continuum (as $f$ is monotone ) and $Y \subset X_{f}$ (as $X_{f}=f^{-1}\left(f\left(X_{f}\right)\right)$.) So $\operatorname{dim} Y \leq \operatorname{dim} X_{f}=1$ and we are done.

Applications. Now we apply the proofs of Theorem 1.1 and Proposition 2.1 to some special 2-dimensional continuum $X$ and special maps $g$.

Let $X$ be a 2-dimensional hereditarily indecomposable continuum embeddable in $\mathbb{R}^{3}$. Let $s=\left(s_{1}, s_{2}, s_{3}\right): X \longrightarrow \mathbb{R}^{3}$ be an embedding of $X$ where $s_{i}: X \longrightarrow \mathbb{R}$. Note that for every $i$ each fiber of $s_{i}$ is a planar compactum.

For Theorem 1.1 define $g=\left(g_{1}, g_{2}\right)$ by $g_{1}=s_{1}, g_{2}=s_{2}$. The fibers of $g$ are embeddable in $\mathbb{R}$ and hence they are 0 -dimensional since $X$ is hereditarily indecomposable. So $\operatorname{dim} g=0$.

For Proposition 2.1 define $g=s_{1}$. The fibers of this map are embeddable in $\mathbb{R}^{2}$ and hence they are of $\operatorname{dim} \leq 1$ as $X$ is hereditarily indecomposable. So in this case $\operatorname{dim} g=1$.

(i) In the proof of Theorem 1.1 we get that for every $i=1,2, \operatorname{dim} p_{i}^{*}=2$. Indeed, take some $i$ and let $K_{1}, K_{2} \in \mathcal{C}(X)$ be such that $K_{1} \neq K_{2}$ and $p_{i}\left(K_{1}\right)=p_{i}\left(K_{2}\right)$. By Proposition $1.3 p_{i}\left(K_{1}\right)$ and $p_{i}\left(K_{2}\right)$ must be singletons and hence $K_{1}, K_{2} \subset$ $p_{i}^{-1}(y)$ for some $y \in Y_{i}$. The fibers of $p_{i}$ are contained in the fibers of $g_{i}$ which can be embedded in the plane (by our special choice of $g_{i}$ ). So by Theorem 1.9 $\operatorname{dim} \mathcal{C}\left(p_{i}^{-1}(y)\right)=2$ and $\operatorname{dim} p_{i}^{*}=2$. 
In particular this implies that for both $Y_{1}$ and $Y_{2}$ we have $\operatorname{dim} \mathcal{C}\left(Y_{i}\right)=\operatorname{dim} \mathcal{C}_{\epsilon}\left(Y_{i}\right)$ $=\infty$ for every $\epsilon>0$ (since $\operatorname{dim} \mathcal{C}_{\epsilon}(X)=\infty$ for every $\epsilon>0$.)

(ii) In the proof of Proposition 2.1 we get that for a sufficiently small $\epsilon>0$, $\operatorname{dim} \mathcal{C}_{\epsilon}(Y)=2$ (though $\operatorname{dim} \mathcal{C}(Y)=\infty$ !).

Define $t=\inf \left\{\operatorname{diam} f^{-1}(f(y)): y \in Y\right\} . t>0$ since $f$ has non-trivial fibers. Note that $f^{-1}(f(y))$ is contained in $Y$ for every $y \in Y$. Take any open monotone map $d$ on $Y$ such that $r=\sup \left\{\operatorname{diam} d^{-1}(d(y)): y \in Y\right\}<t$. Let us show that $\operatorname{dim} d(Y)=1$. Take some $y \in Y$. Since $Y \subset X_{f}$ we have $q^{-1}(q(y))=f^{-1}(f(y))=$ $f^{-1}(f(y)) \cap p^{-1}(p(y))$. So $f^{-1}(f(y))$ is contained in some fiber of $p$ and hence $f^{-1}(f(y))$ is also contained in some fiber of $g$ which is planar (by our choice of $g)$. By Proposition 1.3 the restriction of $d$ to the continuum $f^{-1}(f(y))$ is open and monotone and hence by Theorem $1.9 \operatorname{dim} d\left(f^{-1}(f(y))\right) \leq 1$ and since $r<t$ we have $\operatorname{dim} d\left(f^{-1}(f(y))\right)=1$. So $d(Y)$ is covered by 1 -dimensional continua and hence by Theorem $1.8 \operatorname{dim} d(Y)=1$.

Let $W$ be a Whitney map for $Y$, let $a>0$ be such that for $F \in \mathcal{C}(Y), W(F) \leq a$ implies that $\operatorname{diam} F<t$ and let $\epsilon>0$ be so small that $\mathcal{C}_{\epsilon}(Y) \subset W^{-1}([0, a])$. Then $\operatorname{dim} W^{-1}(b)=1$ for $0 \leq b \leq a$ since $W^{-1}(b)$ is an image of $Y$ under an open monotone map with fibers of diam $<t$ and by the above such an image is of $\operatorname{dim}=1$. Hence by Hurewicz's theorem $\operatorname{dim} W^{-1}([0, a]) \leq 2$. On the other hand for every non-trivial $F \in \mathcal{C}_{\epsilon}(Y), \mathcal{C}(F)$ is embeddable into $\mathcal{C}_{\epsilon}(Y)$ and by [9], Theorem 2.1, $\operatorname{dim} \mathcal{C}(F) \geq 2$. So $\operatorname{dim} \mathcal{C}_{\epsilon}(Y)=2$ and we are done.

(iii) In the proof of Proposition 2.1 we get that $\operatorname{dim} f(Y)=2$ (this should be compared with Theorem 1.2).

We recall that for every $z \in q\left(X_{p}\right), z=q_{p}^{-1}\left(q_{p}(z)\right)$.

Let $z \in q\left(X_{f}\right)$. It is easy to see that $q_{p}^{-1}\left(q_{p}(z)\right) \subset q\left(X_{f}\right)$ and $q_{f}\left(q_{p}^{-1}\left(q_{p}(z)\right)\right)=$ $f\left(p^{-1}\left(q_{p}(z)\right)\right)$. By Proposition 1.3 the restriction of $f$ to $p^{-1}\left(q_{p}(z)\right)$ is open and monotone and since $p^{-1}\left(q_{p}(z)\right)$ is a planar continuum, $\operatorname{dim} f\left(p^{-1}\left(q_{p}(z)\right)\right) \leq 1$ by Theorem 1.9, and as $q_{f}$ is a homeomorphism on $q\left(X_{f}\right)$ we have

$$
\operatorname{dim} q_{p}^{-1}\left(q_{p}(z)\right)=\operatorname{dim} q_{f}\left(q_{p}^{-1}\left(q_{p}(z)\right)\right)=\operatorname{dim} f\left(p^{-1}\left(q_{p}(z)\right)\right) \leq 1 .
$$

So $\operatorname{dim} q_{p} \leq 1$ and by Hurewicz's theorem $\operatorname{dim} Z \leq \operatorname{dim} p(X)+\operatorname{dim} q_{p} \leq 1+1=2$. Then $\operatorname{dim} f(Y) \leq \operatorname{dim} f\left(X_{f}\right)=\operatorname{dim} q\left(X_{f}\right) \leq \operatorname{dim} Z \leq 2$ and hence $\operatorname{dim} f(Y)=$ $\operatorname{dim} Z=2$ as $\operatorname{dim} f(Y) \geq \operatorname{dim} X=2$.

Proof of Theorem 1.2. Replacing $X$ by a 2-dimensional subcontinuum of $X$ and applying Proposition 1.3 we may assume without loss of generality that $\operatorname{dim} X=2$.

Then there exist disjoint closed subsets $F_{1}, F_{2}$ of $X$ and $r=r\left(F_{1}, F_{2}\right)>0$ such that every closed set which separates between $F_{1}$ and $F_{2}$ has a component of $\operatorname{diam} \geq r$. Assume that

(1) the fibers of $\varphi$ are of diam $<\min \left\{r\right.$, dist. $\left.\left(F_{1}, F_{2}\right)\right\}$.

Let $W: \mathcal{C}(X) \longrightarrow \mathbb{R}^{+}$be a Whitney map for $X$. Since the fibers of $\varphi$ are nontrivial we can take some $0<t<\inf \left\{W\left(\varphi^{-1}(y)\right): y \in \varphi(X)\right\}$ and let $f: X \longrightarrow$ $f(X)=W^{-1}(t)$ be the quotient map. For every $x \in X, \varphi^{-1}(\varphi(x))$ is not contained in $f^{-1}(f(x))$ since $W\left(f^{-1}(f(x))\right)=t<W\left(\varphi^{-1}(\varphi(x))\right)$ and as $X$ is hereditarily indecomposable we have $f^{-1}(f(x)) \subset \varphi^{-1}(\varphi(x))$ and $f^{-1}(f(x)) \neq \varphi^{-1}(\varphi(x))$. Then there exists $\psi: f(X) \longrightarrow \varphi(X)$ such that $\varphi=\psi \circ f$ and clearly $f$ and $\psi$ are open monotone maps with non-trivial fibers.

We will use the construction, notations and conclusions of the proof of Proposition 2.1 for $f$ and some auxiliary 1-dimensional map $g: X \longrightarrow \mathbb{R}$. 


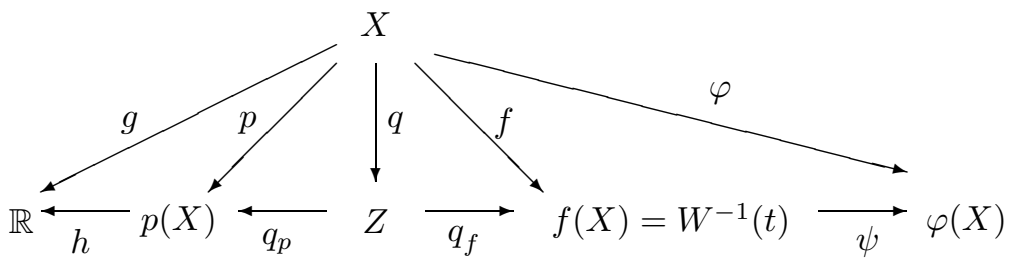

Recall that in the end of the proof of Proposition 2.1 we define $Y=f^{-1}(T)$ where $T$ is a component of $f\left(X_{f}\right)$ which satisfies the condition $\operatorname{dim} T \geq 2$. For Theorem 1.2 we need that $T$ satisfies another condition instead of $\operatorname{dim} T \geq 2$, namely:

(2) for every closed $L$ which separates between $f\left(F_{1}\right)$ and $f\left(F_{2}\right)$ there exists a component of $f^{-1}(L) \cap f^{-1}(T)$ with diam>r.

Note that by (1) $\varphi\left(F_{1}\right) \cap \varphi\left(F_{2}\right)=\emptyset$ and hence $f\left(F_{1}\right) \cap f\left(F_{2}\right)=\emptyset$. Let us show that there exists a component $T$ of $f\left(X_{f}\right)$ which satisfies $(2)$.

Assume the contrary, that is, for every component $T$ of $f\left(X_{f}\right)$ we can find a closed set $L_{T}$ separating $f\left(F_{1}\right)$ and $f\left(F_{2}\right)$ such that every component of $f^{-1}\left(L_{T}\right) \cap f^{-1}(T)$ is of diam $\leq r$. Then one easily obtains that there exist closed subsets $G_{T}^{1}, G_{T}^{2}$ and $V_{T}$ of $f\left(X_{f}\right)$ such that

(3) $V_{T}$ is clopen in $f\left(X_{f}\right)$,

(4) $f\left(F_{1}\right) \cap V_{T} \subset G_{T}^{1} \subset V_{T}, f\left(F_{2}\right) \cap V_{T} \subset G_{T}^{2} \subset V_{T}$ and $G_{T}^{1} \cap G_{T}^{2}=\emptyset$,

(5) every component of $f^{-1}\left(V_{T} \backslash\left(G_{T}^{1} \cup G_{T}^{2}\right)\right)$ is of diam $\leq r$.

Take a finite cover $V_{T_{1}}, V_{T_{2}}, \ldots, V_{T_{m}}$ of $f\left(X_{f}\right)$ where $T_{1}, T_{2}, \ldots, T_{m}$ are components of $f\left(X_{f}\right)$ and define

$$
\begin{aligned}
& G_{1}=G_{T_{1}}^{1} \cup\left(G_{T_{2}}^{1} \backslash V_{T_{1}}\right) \cup\left(G_{T_{3}}^{1} \backslash\left(V_{T_{1}} \cup V_{T_{2}}\right)\right) \cup \ldots \cup\left(G_{T_{m}}^{1} \backslash\left(V_{T_{1}} \cup \ldots \cup V_{T_{m-1}}\right)\right), \\
& G_{2}=G_{T_{1}}^{2} \cup\left(G_{T_{2}}^{2} \backslash V_{T_{1}}\right) \cup\left(G_{T_{3}}^{2} \backslash\left(V_{T_{1}} \cup V_{T_{2}}\right)\right) \cup \ldots \cup\left(G_{T_{m}}^{2} \backslash\left(V_{T_{1}} \cup \ldots \cup V_{T_{m-1}}\right)\right) .
\end{aligned}
$$

Then by (4) $f\left(F_{1}\right) \cap f\left(X_{f}\right) \subset G_{1} \subset f\left(X_{f}\right), f\left(F_{2}\right) \cap f\left(X_{f}\right) \subset G_{2} \subset f\left(X_{f}\right), G_{1} \cap G_{2}=$ $\emptyset$ and by (3) and (5) we have

(6) every component of $f^{-1}\left(f\left(X_{f}\right) \backslash\left(G_{1} \cup G_{2}\right)\right)$ is of diam $\leq r$.

Let a closed subset $H$ of $Z$ separate $q_{f}^{-1}\left(f\left(F_{1}\right) \cup G_{1}\right)$ and $q_{f}^{-1}\left(\left(f\left(F_{2}\right) \cup G_{2}\right)\right.$ such that $\operatorname{dim} H \cap q\left(X_{p}\right) \leq 0$ (we recall that $\operatorname{dim} q\left(X_{p}\right)=\operatorname{dim} p\left(X_{p}\right) \leq \operatorname{dim} p(X) \leq 1$ ). It is easy to see that each $z \in H \backslash q\left(X_{f}\right)$ is a component of $H$ since $q\left(X_{f}\right)$ is closed and the set $H \backslash q\left(X_{f}\right) \subset H \cap q\left(X_{p}\right)$ is 0-dimensional. Recall that $X_{f}=f^{-1}\left(f\left(X_{f}\right)\right)=$ $q^{-1}\left(q\left(X_{f}\right)\right)$. So for every component $K$ of $q^{-1}(H)$ which intersects $X \backslash X_{f}, q(K)$ intersects $H \backslash q\left(X_{f}\right)$ and hence $q(K)$ is a singleton and by (1) diam $K \leq r$.

By (6) the components of $q^{-1}(H)$ which are contained in $X_{f}$ also have diam $\leq r$. So every component of $q^{-1}(H)$ is of $\operatorname{diam} \leq r$ and since $q^{-1}(H)$ separates between $F_{1}$ and $F_{2}$ we get a contradiction.

This shows that there exists a component $T$ of $f\left(X_{f}\right)$ for which (2) holds and we claim that $\operatorname{dim} \psi(T)=\infty$. As $f$ is monotone, $f(X)$ is hereditarily indecomposable. By (1) and (2) $\psi(T)$ cannot be a singleton and hence by Proposition 1.3 the restriction of $\psi$ to $T$ is an open monotone map with non-trivial fibers. If $\operatorname{dim} \psi(T)<\infty$, then by Theorem 1.10 there exists a 0 -dimensional closed subset $Q$ of $T$ which intersects every fiber of $\psi$ meeting $T$. Take a closed subset $L$ which separates between $f\left(F_{1}\right)$ and $f\left(F_{2}\right)$ such that $L \cap Q=\emptyset$. Then the components of $f^{-1}(L) \cap f^{-1}(T)$ cannot contain a fiber of $\varphi$ and hence they are contained in the 
fibers of $\varphi$ since $X$ is hereditarily indecomposable. So by (1) the components of $f^{-1}(L) \cap f^{-1}(T)$ have diam $<r$ and this contradicts (2).

Thus $\operatorname{dim} \psi(T)=\infty$ and we obtain that for $Y=f^{-1}(T), \operatorname{dim} \varphi(Y)=\operatorname{dim} \psi(T)$ $=\infty$. Recall that by the proof of Proposition $2.1 \operatorname{dim} Y=1$.

Remark. Combining Theorem 1.2 and Theorem 1.9 we get that every 2-dimensional hereditarily indecomposable continuum (and so every 3-dimensional continuum) contains a 1-dimensional subcontinuum not embeddable in the plane.

\section{REFERENCES}

1. R. H. Bing, Higher-dimensional hereditarily indecomposable continua, Trans. AMS, 71(1951), 267-273. MR 13:265c

2. R. Engelking, Dimension Theory, PWN, Warszawa, 1978. MR 58:2753b

3. J. L. Kelley, Hyperspaces of a continuum, Trans. AMS, 52(1942), 22-36. MR 3:315b

4. J. Krasinkiewicz, On the hyperspaces of certain plane continua, Bull. Pol. Acad. Sci., 23(1975), 981-983. MR 58:12942

5. M. Levin and Y. Sternfeld, Monotone basic embeddings of hereditarily indecomposable continua, Top. and Appl., 68 (1996), no. 3, 241-249. CMP 96:09

6. M. Levin and Y. Sternfeld, Hyperspaces of two-dimensional continua, Fundamenta Math., 150 (1996), no. 1, 17-24. CMP 96:12

7. M. Levin and Y. Sternfeld, The space of subcontinua of a 2-dimensional continuum is infinite dimensional, Proceedings AMS, to appear.

8. W. Lewis, Monotone maps of hereditarily indecomposable continua, Proc. AMS, 75(1979), 361-364. MR 82g:54051

9. S. B. Nadler Jr., Hyperspaces of sets, Marcel Dekker, 1978. MR 58:18330

Department of Mathematics, Haifa University, Mount Carmel, Haifa 31905, Israel

E-mail address: levin@mathcs2.haifa.ac.il

Current address: Department of Mathematics, University of Washington, Box 354350, Seattle, Washington 98195-4350

E-mail address: levin@math.washington.edu 